\title{
Changes Produce during Apple Refrigeration Storage in Textural and Structural Fruit Behavior
}

\author{
Oana Viorela Nistor, Elisabeta Botez, Gabriel Dănuț Mocanu and Doina Georgeta Andronoiu \\ Department of Bioengineering in Food Industry, Faculty of Food Science and Engineering, “Dunarea de Jos” University, Galati \\ 800201, Romania
}

Received: August 19, 2011 / Published: June 20, 2012.

\begin{abstract}
The purpose of this study was to determine from the three different varieties of apple (Golden Delicious, Jonathan and Starkrimson), which is best suited to storage at refrigeration temperature in constant conditions $\left(5{ }^{\circ} \mathrm{C}\right.$ and $85 \%$ relative humidity). In this order, the apples were stored in three variants of packages as bulk, packed in LDPE foil and alimentary paper for 23 days in a professional refrigerator. In this period, some textural and structural tests based on penetration and microstructure analyses were carried out with a 7-day frequency. As a conclusion of these tests the maximum values for mechanical determination are for Starkrimson variety $69.54 \mathrm{~N}$ at $4 \mathrm{~mm}$ depth for 15 days of storage while the minimum one is $24.34 \mathrm{~N}$ at $3 \mathrm{~mm}$ for Jonathan variety. The good part of the storage by refrigeration is the bulk variants there were no significant changes including quality damage, even the Starkrimson variety microstructure modification during refrigeration was the most affected.
\end{abstract}

Key words: Apple, texture, structure, bulk, LDPE foil, alimentary paper, penetrometer, epifluorescence microscope.

\section{Introduction}

Cultivated apple (Malus domestica) is among the most diverse and ubiquitously cultivated fruit species. Apple is a member of the Rosaceae family, which includes many commercial fruit (e.g., pear (Pyrus communis), strawberry (Frageria spp.), cherry (Prunus avium), peach/nectarine (Prunus persica), apricot (Prunus armeniaca)), nut (almond (Prunus amygdalus)), forest (black cherry (Prunus serotina)), and ornamental (rose (Rosa hybrida)), crab apple (Malus coronaria)) species [1].

Like tomato, apple is a climacteric fruit, with a clear respiratory climacteric and ethylene peak associated with ripening. However, unlike tomato, which is a true berry fruit, the majority of apple fruit is derived from proliferated receptacle tissues, with the ovary-derived tissues restricted to the center of the

Corresponding author: Oana Viorela Nistor, Ph.D. candidate, assistant professor, research field: food engineering. E-mail:Oana.Nistor@ugal.ro. mature fruit (core). The skin (epidermal and subepidermal cell layers) is strikingly different from the cortex, and, in almost all apple varieties studied thus far, biosynthesis of pigments and most volatile esters associated with aroma is concentrated in epidermal and subepidermal tissues. Pigmentation is dominated by anthocyanins (compared with carotenoids in tomato), and tomato fruit is not known to synthesize volatile esters. Compared with most fruits, apple has an extremely long developmental sequence, often exceeding $150 \mathrm{~d}$. Although ripening in apple is accompanied by changes in texture, it is one of the few commercially important fruits that undergo significant softening only after extended storage and deterioration.

An additional attraction of apple for studies of fruit biology is the enormous diversity in fruit-related traits among the large number of cultivars and related wild genotypes $(3,000)$ available for analysis.

Deterioration of fruits and vegetables during storage depends largely on temperature. One way to slow 
down this change and so increase the length of time fruits and vegetables can be stored, is by lowering the temperature to an appropriate level. It must be remembered that if the temperature is too low the produce will be damaged and also that as soon as the produce leaves the cold store, deterioration starts again and often at a faster rate [2].

Temperature is the most important environmental factor that influences the preservation of fruits harvested. Deterioration is mediated through the effect of temperature on the physiological and biochemical processes that occur in fruit harvested.

Lowering the temperature influences all metabolic processes governed by enzymes. Temperature will influence the activity of the enzymes catalyzing the biochemical reactions maintaining homeostasis in cells. However, these enzyme systems can be affected differentially by temperature, why this can lead to imbalances in metabolism [3].

Relative humidity is the most common way of expressing the amount of moisture in air. The difference in the amount of moisture between the air in the fruits and around the fruit determines the driving force for water loss from fruits harvested. When at a given temperature, the relative humidity of the air around the fruits increases, the driving force is reduced rapidly. However, even at $100 \%$ RH (saturated conditions), there may be some water loss, because the fruits temperature is unlikely to equal the air temperature owing to heat of respiration [4].

Airflow around fruits affects relative humidity, temperature, and gas composition. Uneven or inadequate airflow will result in a wide spread of product temperatures throughout unit loads (i.e., packages or pallets of product), inducing spatial variation in product quality and subsequent shelf life. Sufficient air movement is necessary to remove heat of respiration and to minimize temperature gradients; but the higher the flow rates, the higher the rates of water loss from the produce (by reduced RH). The optimum airflow will be a compromise between removing heat from and maintaining water in harvested vegetables [5].

Postharvest handling and storage systems influence the quality of harvested fruits by affecting the physiology of fruits harvested. Changes in the physiology of harvested vegetables are mediated through the influence of the environmental conditions created by the handling and storage system. The physiology of the harvested fruits, environmental conditions, and handling and storage systems all interact and therefore cannot be considered in isolation when successfully preserving and storing high quality fruits harvested.

Processing technologies being used to ensure food quality and safety are freezing; thermal processing, sterilization, drying, refrigeration, and the controlled distribution of fresh produce [6]. In addition to taste, texture is a quality attribute that is critical in determining the acceptability of apple fruits by consumers. Fruit wholesalers are therefore particularly interested in the measurement of fruit texture. However, direct measurement of texture through sensory analysis is very complex and time consuming. For this reason, many attempts have been made to replace sensory analysis with instrumental measurements [7].

The correlation coefficients obtained between sensory texture attributes and penetrometric force were very different from one study to another as a function of cultivars, ripening stages, global fruit evolution during storage, fruit origin, etc. [8].

The present paper has the aim to demonstrate the inherent modifications appeared during the refrigeration storage. As principal objectives of the changes produce during apple refrigeration storage regarded the textural and structural transformations and their comparison. For reaching these objectives there were used some instrumental methods to determine the textural and structural modifications.

\section{Materials and Methods}

For the study there were used three varieties of 
apple (Starkrimson, Jonathan and Golden Delicious) and two types of package material alimentary paper and LDPE (Low Density Polyethylene) foil adding also the bulk variant, lancet.

For refrigeration it was used a professional refrigerator with temperature and humidity sensor and to determine the changes produced during storage there were used an epifluorescence microscope and a penetrometer (Fruit texture analyzer).

The apples were stored at $5{ }^{\circ} \mathrm{C}$ for 23 days. There were determined: apples' texture and structure using a 7-day analysis interval.

To determine the apples texture variation it was used a penetrometer which is applying a force over some apple's parts measuring the necessary force for puncture the fruit. For this analysis, whole apples were used. A three penetration procedure in three different anatomical points of the apple is used for good result. So this is the base for the curves generated by the computer regarding the firmness, an important texture quality.

For structure determination it was chosen an epifluorescence microscope which can see the microstructure modifications only if the samples are cut at a spangle size.

\section{Results and Discussion}

\subsection{Bulk and Packed Starkrimson Variety Storage Structural Modifications}

After the first period of storage that was analyzed the general appearance of the apples by visual observing. Starkrimson variety structure variation during storage is showed in Fig. 1 also for the bulk as for the packed apples. At this stage presented in Fig. 1 the apples are firm, with soft skin and juicy pulp for all apples varieties.

Now, it is important to show if there are some differences between the visual findings and the measurements one.

In all images it can be seemed the starch congestions, the filaments of pectin which are immersed in the water mass, also another important aspect to observe is that the proportion between the principal components from the three apple variants are different.

After the storage, the Starkrimson variety alimentary paper packed were the most affected. The Starkrimson apple contains especially starch than pectin.

The sugars from the parenchyma tissues can be observed in the Fig. 1, in which the macromolecules are represented by the starch, micromolecules-phenols, respectively fibrous chains can be associated to pectin.

\subsection{Bulk and Packed Starkrimson Variety Storage Textural Modifications}

The puncture test is a simple and easy way to test puncture force that gives an idea about the hardness/stiffness of the solid samples. It is also a good and simple way to test food materials with heterogeneous structure (e.g., cereal bars, chocolates with various layers, etc.) [9].

For Starkrimson variety the firmness of the bulk sample is presented in Fig. 2, where it can be see at a $4 \mathrm{~mm}$ depth the firmness value is $55.46 \mathrm{~N}$, while after other 7-day of storage the necessary force for apple penetration is $60.79 \mathrm{~N}$ at $4 \mathrm{~mm}$.

As much as the depth is growing $(6-7 \mathrm{~mm})$ the penetration force is decreasing $(29.5 \mathrm{~N}, 22 \mathrm{~N})$, this action may be due to the composition of the first layer of the apple wax and pectin rich, which is a resistance force opponent to penetration plunger. Also it can be observed that after a specific depth as 6-7 $\mathrm{mm}$ the curves are almost constant that can be due to the pulp layer where the air globules and the chemical compounds are in perfect balance.

Opposite to the expectations, after the storage the firmness values were not decreasing, without any large variation the values being appropriate as for the alimentary paper packed after seven days of storage the penetration force is $57.31 \mathrm{~N}$ for $3 \mathrm{~mm}$, while at 15 days of storage the force is $58.84 \mathrm{~N}$ at $3 \mathrm{~mm}$ and for the LDPE foil. 


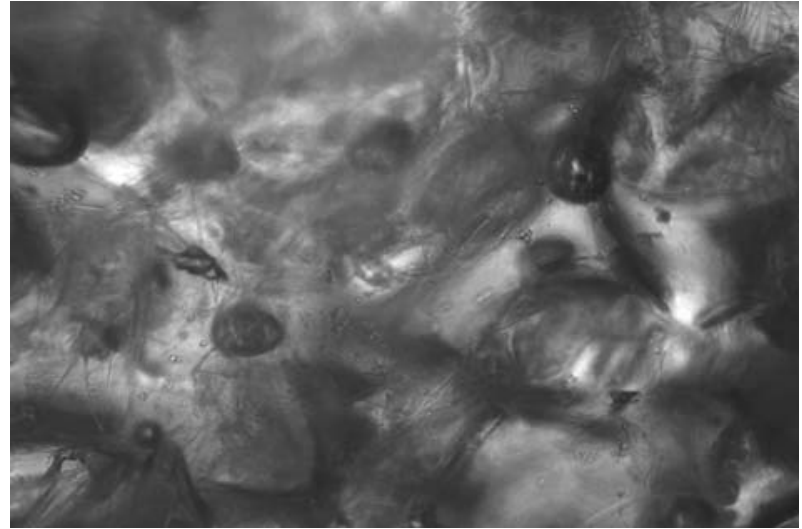

a. aulk apple structure after seven days of storage

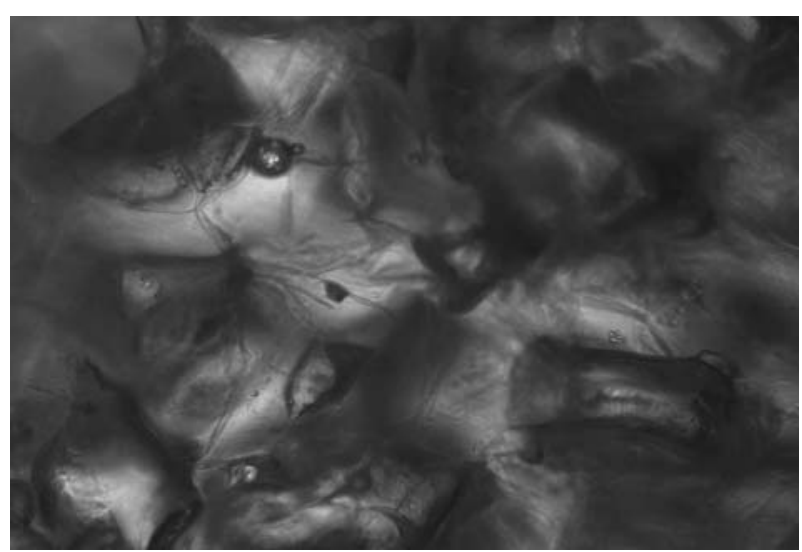

c. alimentary paper packed apple after seven days of storage

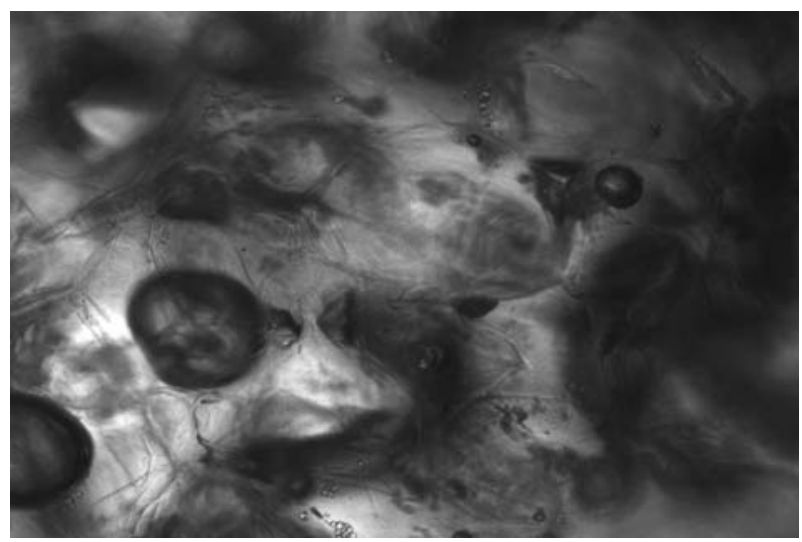

e. LDPE foil packed apple after seven days of storage

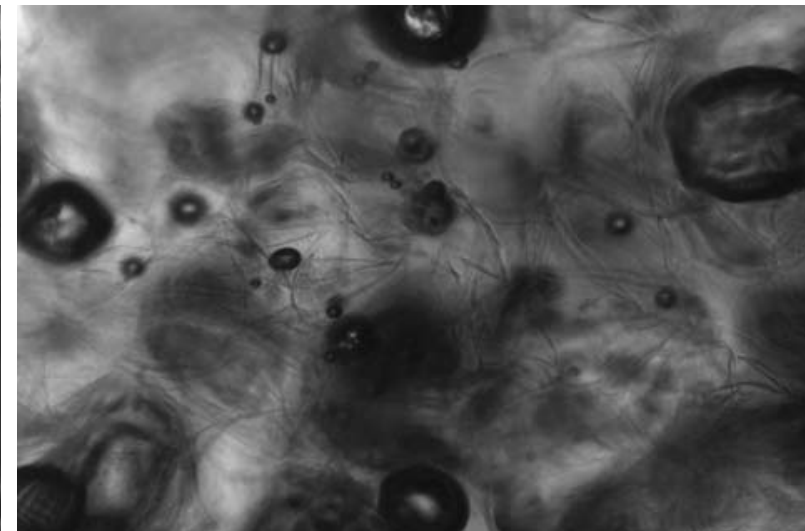

b. bulk apple structure after 15 days of storage

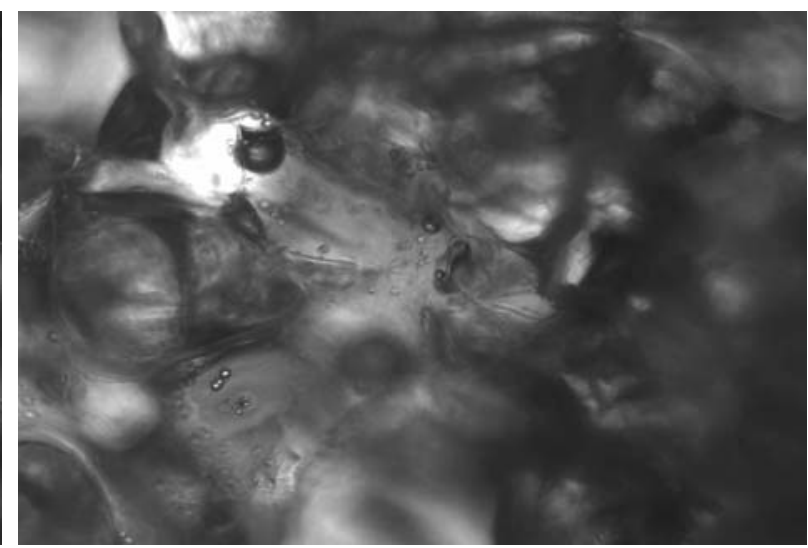

d. alimentary paper packed apple after 15 days of storage

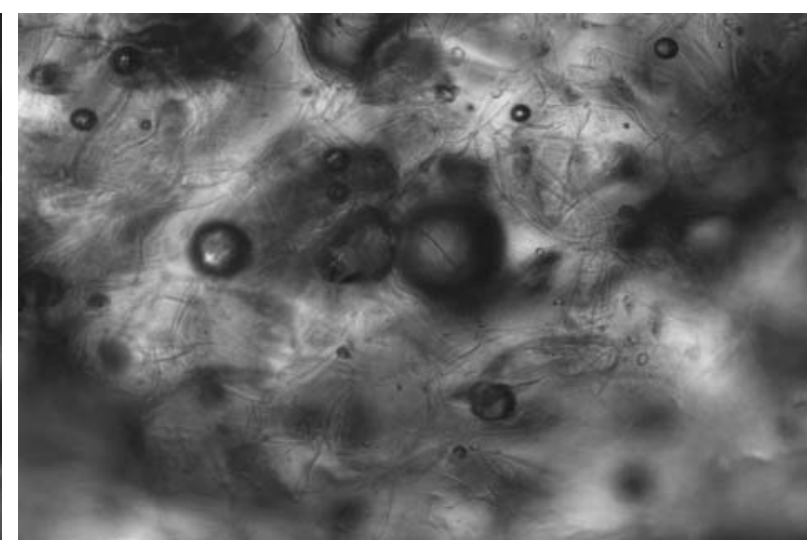

f. LDPE foil packed apple after 15 days of storage

Fig. 1 Starkrimson variety structure variation during storage.

As a conclusion the Starkrimson variety firmness stored at refrigeration temperature is not affected, this result may be due to the sensorial apple properties like mastication, juiciness and lubricity.

Regarding the Jonathan behavior during storage is presented in Fig. 3 as the structure evolution during refrigeration and the modifications produces after seven and 15 days of refrigeration.

From Fig. 3 it can be observed that the Jonathan variety contains a big quantity of phenol comparing with Starkrimson and it has remarked more transformation in bulk variant as it is degraded faster than the packed one. 


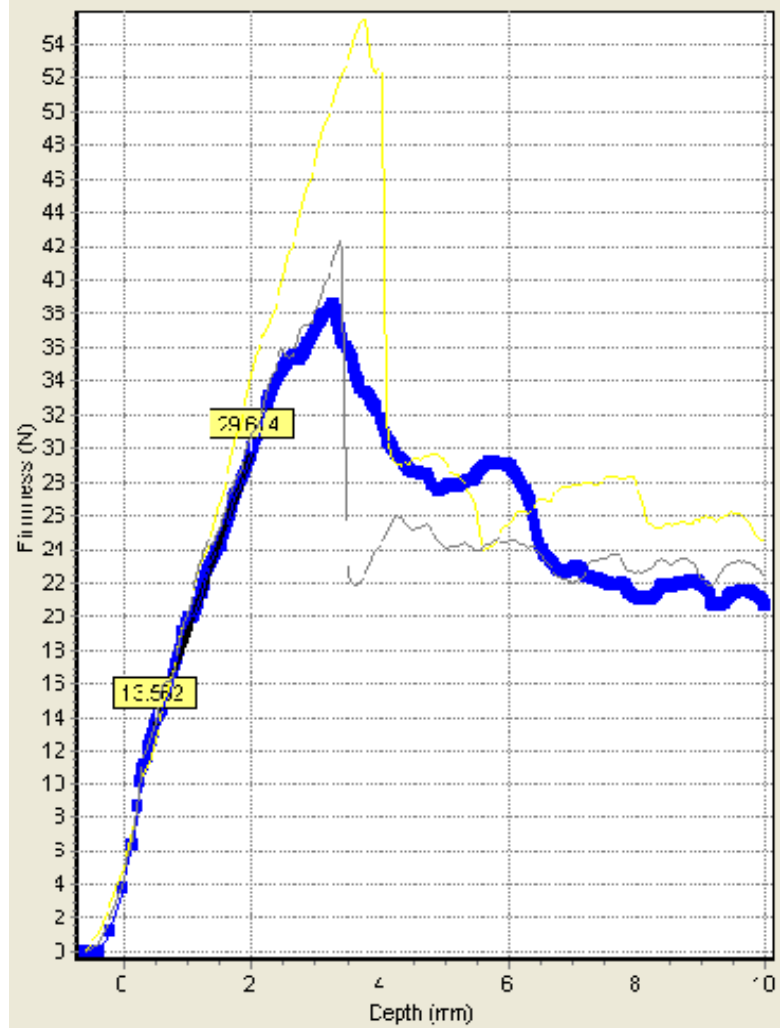

a. apple texture after seven days of storage

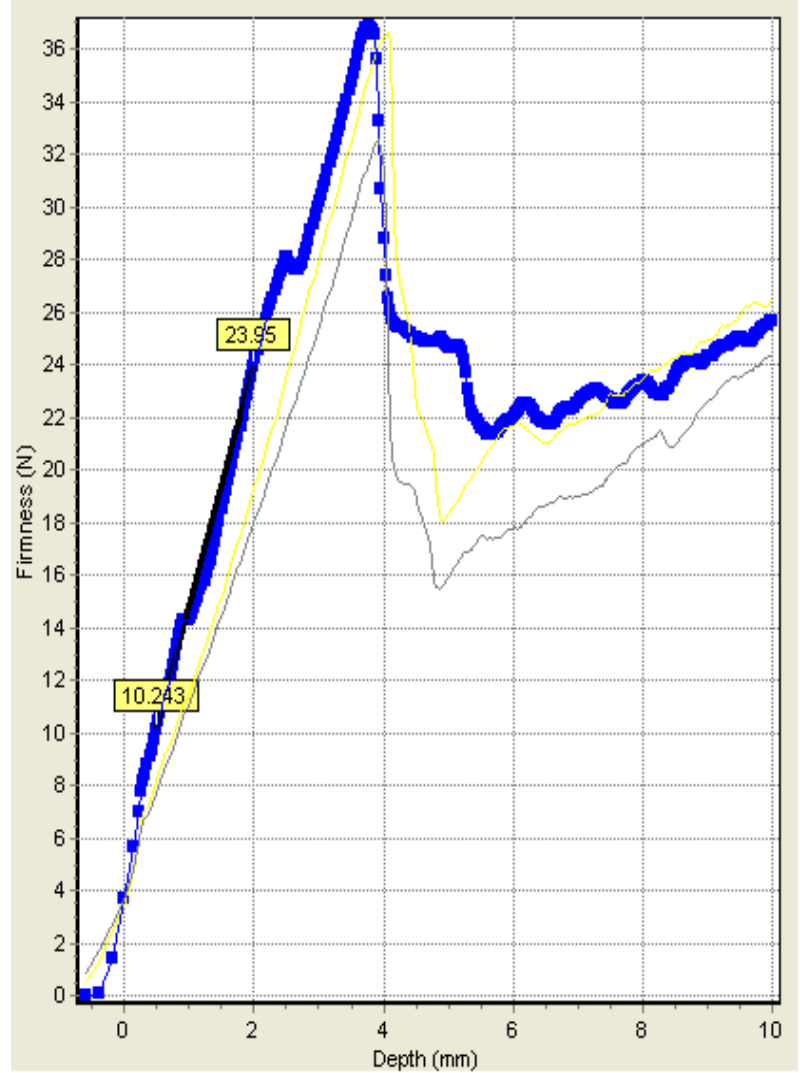

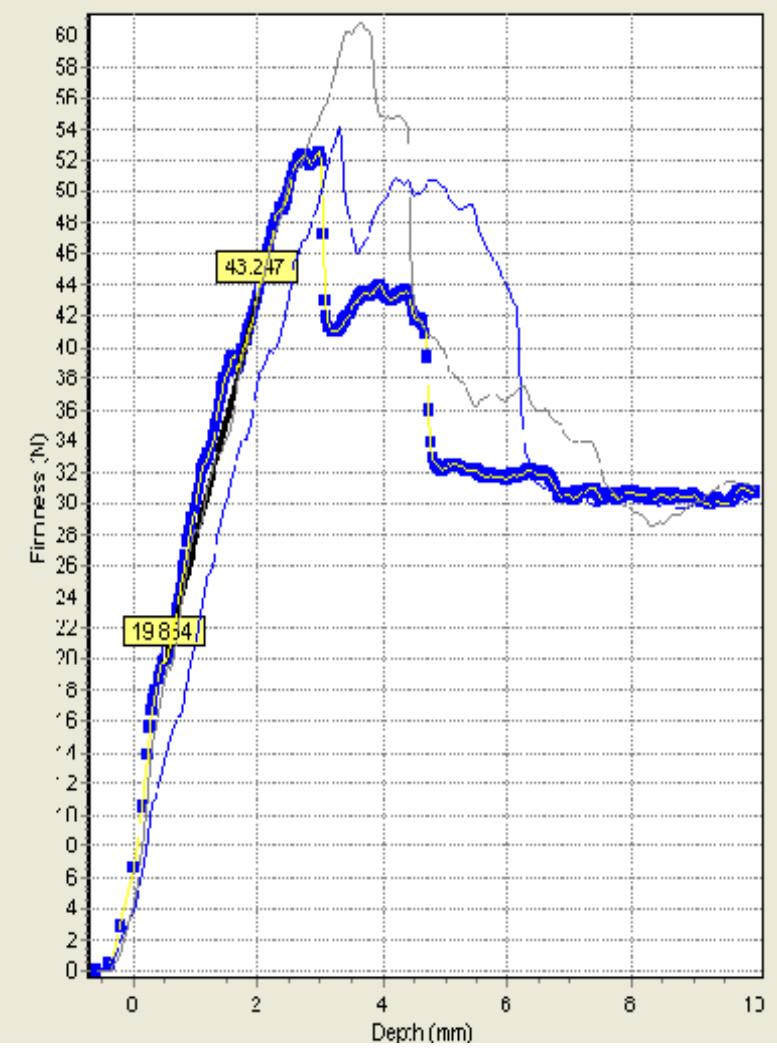

b. apple texture after 15 days of storage

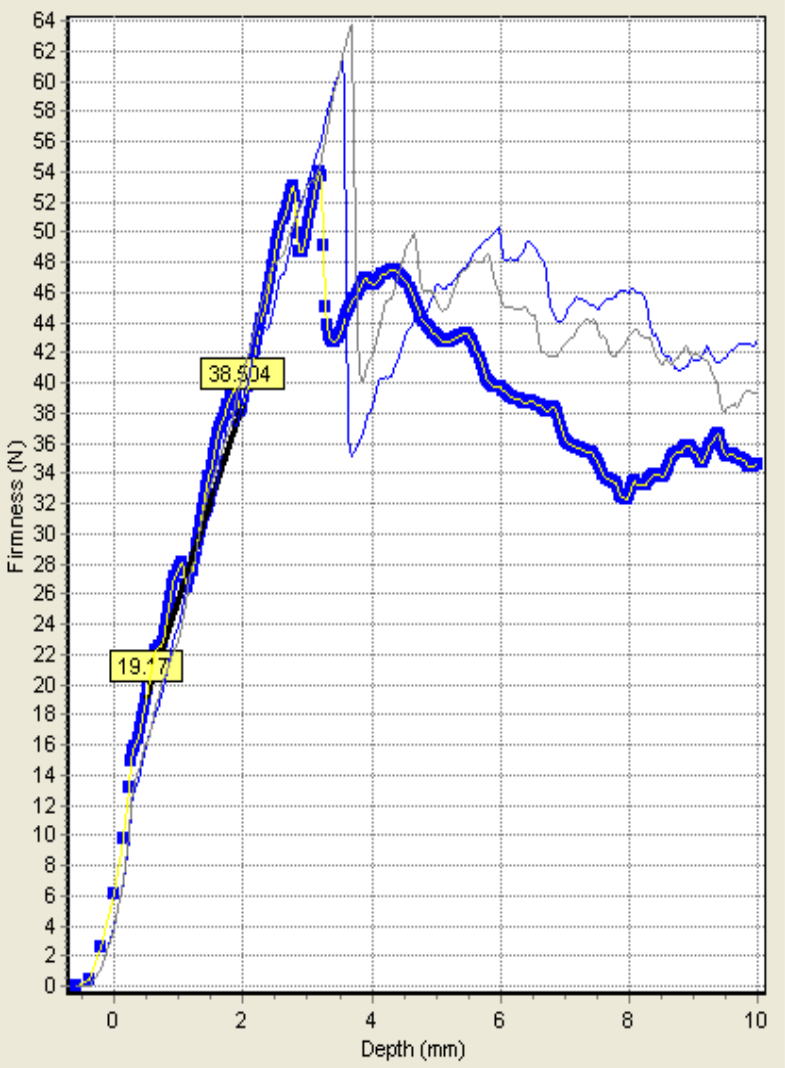

c. alimentary paper packed apple texture after seven days of storage d. alimentary paper packed apple texture after 15 days of storage 


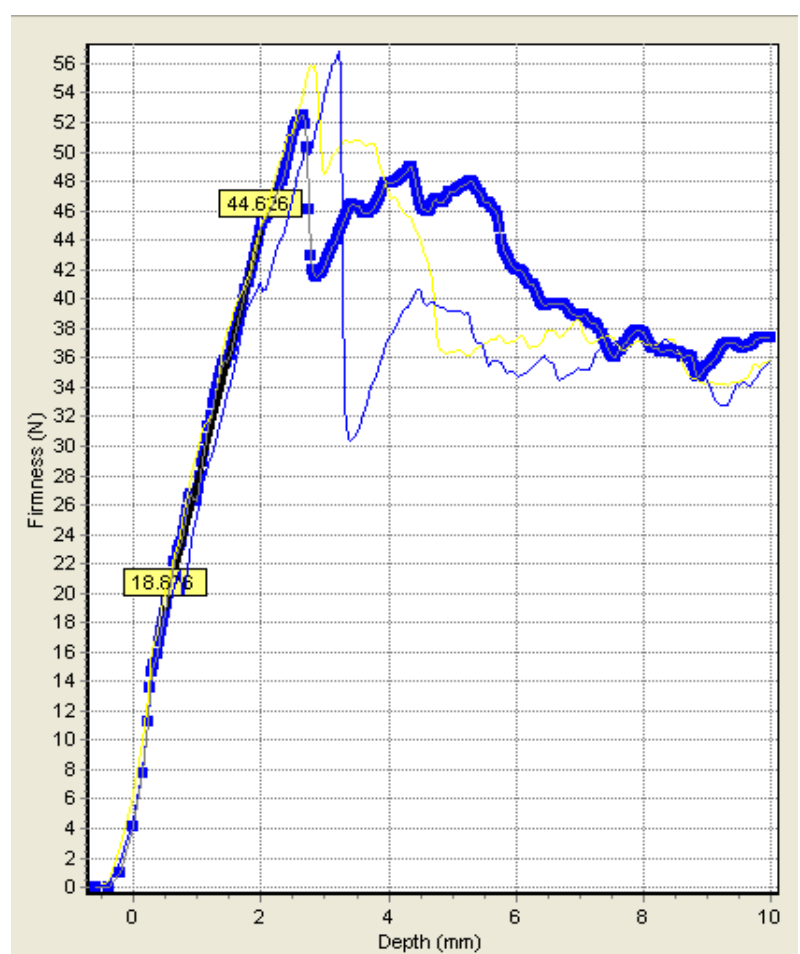

e. LDPE foil packed apple texture after seven days of storage

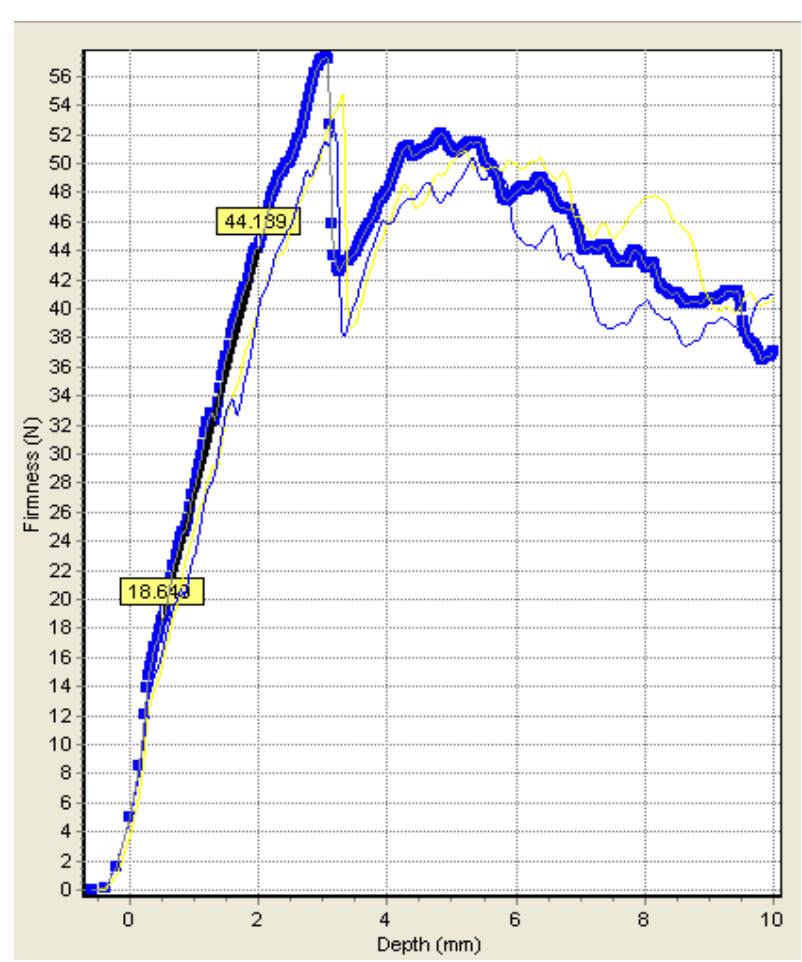

f. LDPE foil packed apple after 15 days of storage

Fig. 2 Starkrimson variety texture curves during storage.

The natural preservatives present naturally in fruit composition as organic acids, flavour and colour compounds are often used in combination with other methods of preservation such as refrigeration, freezing, and dehydration to obtain better control of deleterious organisms. Certain important preservatives such as organic acids and sulfites are multifunctional and their functionalities include preservation as well as antioxidation and antibrowning activities [10].

The same results were obtained for the Jonathan apple variety also by the researcher Thompson [11].

The microstructure modification are similar in the alimentary paper packed case and LDPE foil too, as for the both situation the pectin chains are more visible comparing with the Starkrimson variety.

The Golden Delicious variety contains a bigger water quantity compared with the other two variants. The starch is present in a reduced quantity compared with the high pectin and phenol contain.

After the storage, the microstructure of the bulk apples are affected, being followed by the alimentary paper packed, the best behaviour is registered by the LDPE foil package.

The texture of the Golden Delicious variety is determined by the curves from the graphics showed in Fig. 4 generated by applying a force over the apple at different depths.

The first determination for the bulk sample had registered a $54.54 \mathrm{~N}$ for a $4 \mathrm{~mm}$ depth, the next determinations results being in decreasing at higher depth. At 15 days of storage the force value is $45.33 \mathrm{~N}$ at $4 \mathrm{~mm}$. The firmness is just a little bit affected by storage. These results may be due to the higher quantity of air from the intercellular space or to the water higher quantity from the Golden Delicious variety.

As for the bulk variant for the alimentary paper and LDPE package the firmness results are similar, so for the first type of package at $4 \mathrm{~mm}$ the force value is $43.20 \mathrm{~N}$, for 15 days of storage $49.99 \mathrm{~N}$ for the same depth, while for the other type of package at seven days of refrigeration the force value is 41.05 at $3 \mathrm{~mm}$ and at 15 days of storage $45.33 \mathrm{~N}$ at $4 \mathrm{~mm}$ depth. 


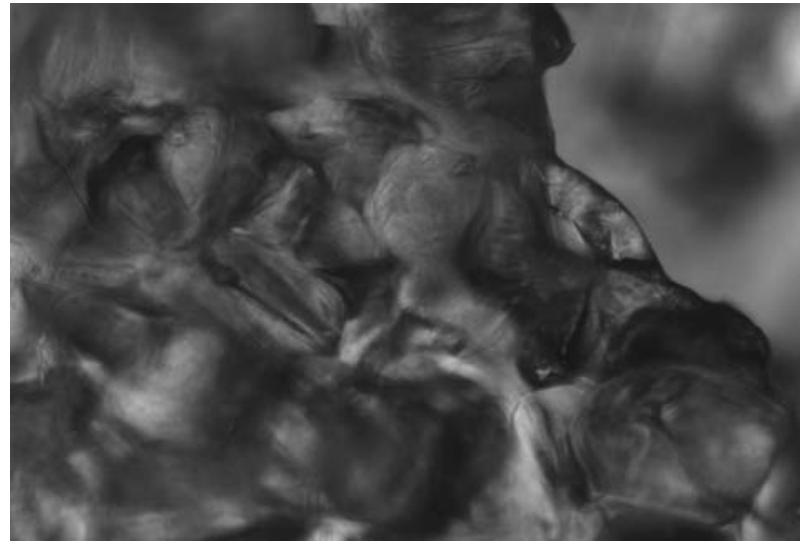

a. bulk apple structure after seven days of storage

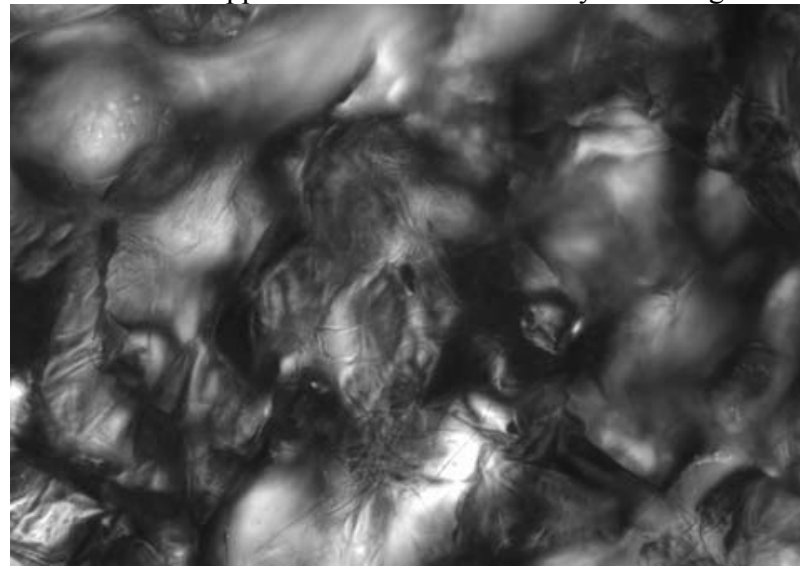

c. alimentary paper packed apple after seven days of storage

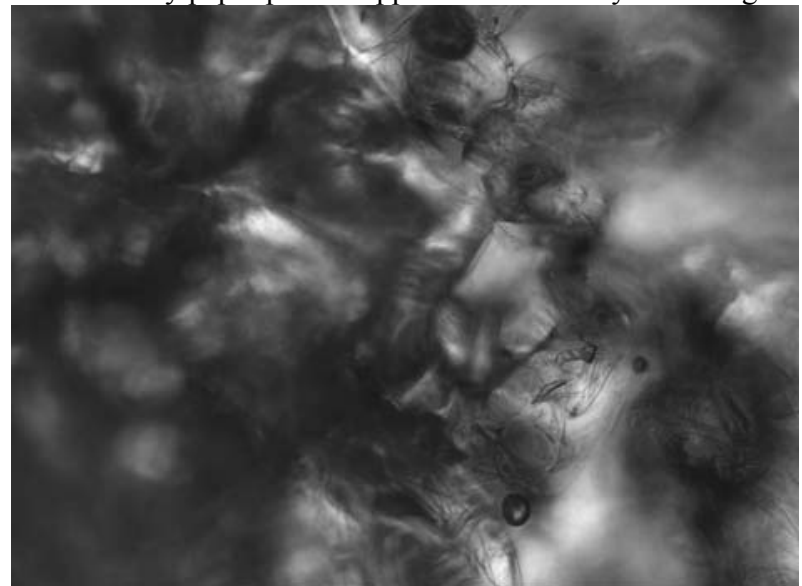

e. LDPE foil packed apple after seven days of storage

Fig. 3 Jonathan variety structure variation during storage.

So as a conclusion the alimentary paper and also the LDPE foil are suitable for the Golden Delicious variety storage.

For the Jonathan variety, it had been registered the $24.34 \mathrm{~N}$ at $3 \mathrm{~mm}$ for seven days of storage in bulk variant and $33.39 \mathrm{~N}$ at $3 \mathrm{~mm}$, while for the packed variants, the force for the alimentary paper packed

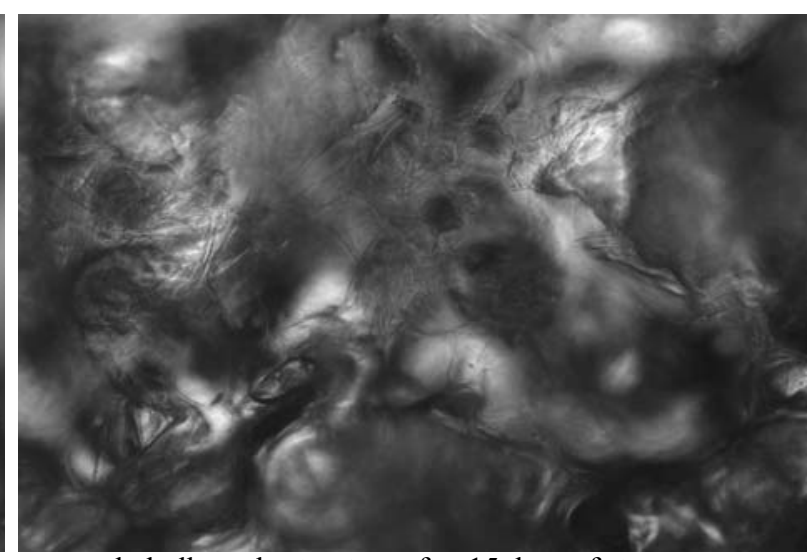

b. bulk apple structure after 15 days of storage

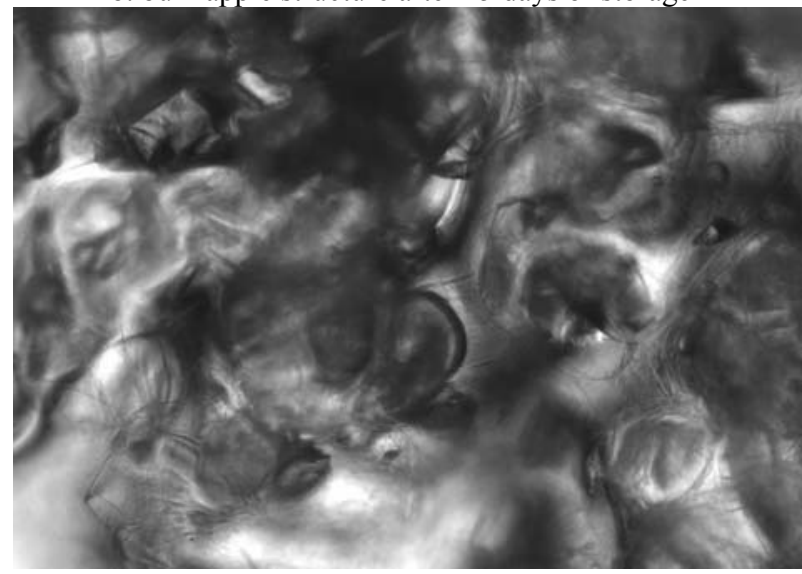

d. alimentary paper packed apple after 15 days of storage

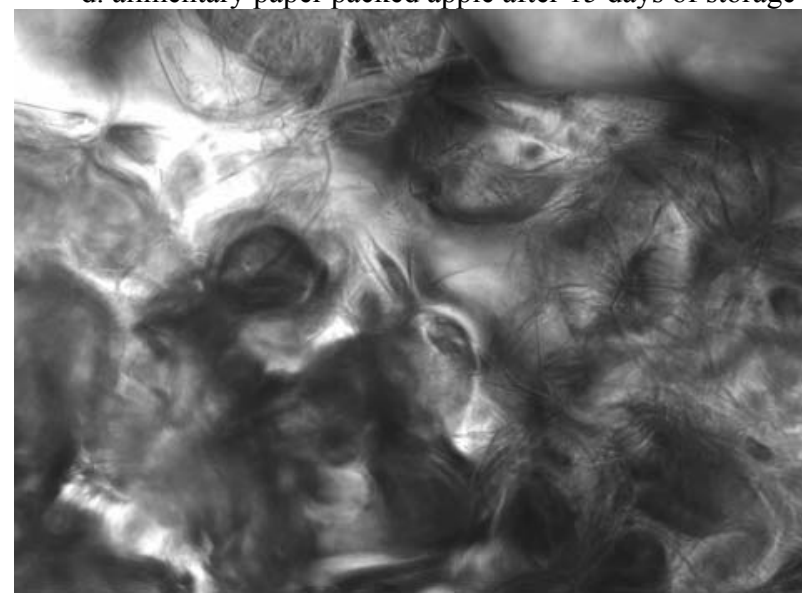

f. LDPE foil packed apple after 15 days of storage

apple is $43.20 \mathrm{~N}$ at $4 \mathrm{~mm}$ and $38.39 \mathrm{~N}$ at $3 \mathrm{~mm}$ for the 15 days stored, for the LDPE packed, the force value is $34.12 \mathrm{~N}$ at $3 \mathrm{~mm}$ and $38.87 \mathrm{~N}$ at $4 \mathrm{~mm}$.

The firmness changes are not so big, but there is a decreasing value for the alimentary paper packed apple, which involves that this is not a suitable type of package for this apple variety. 


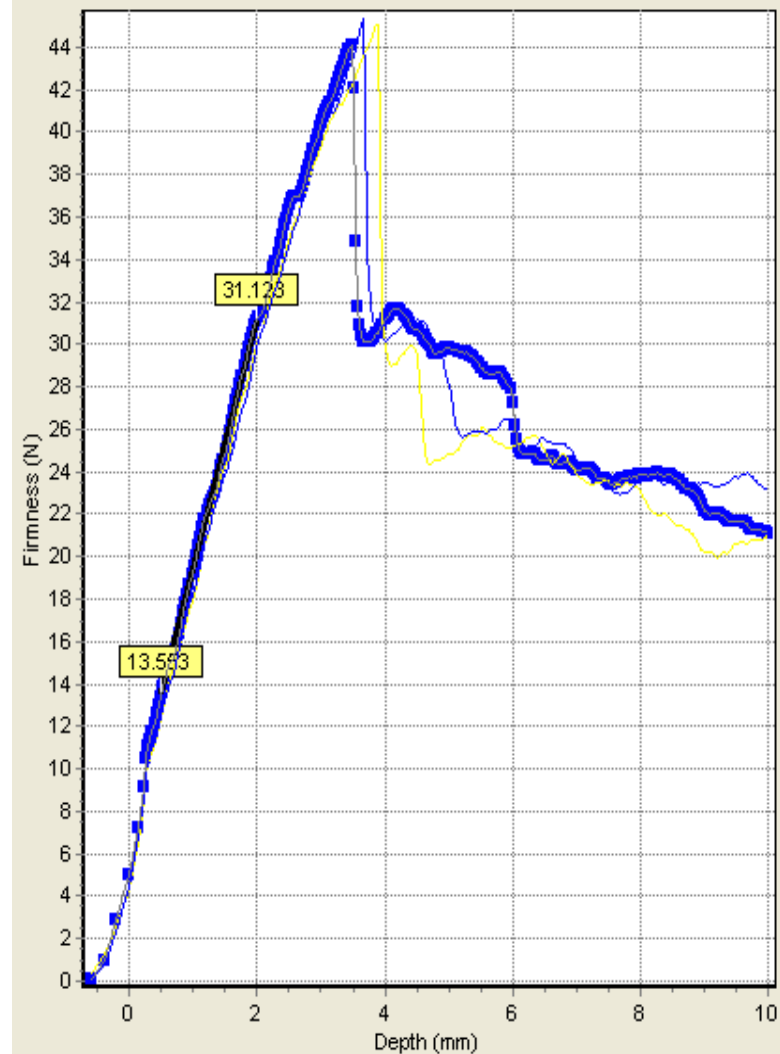

a. apple texture after seven days of storage

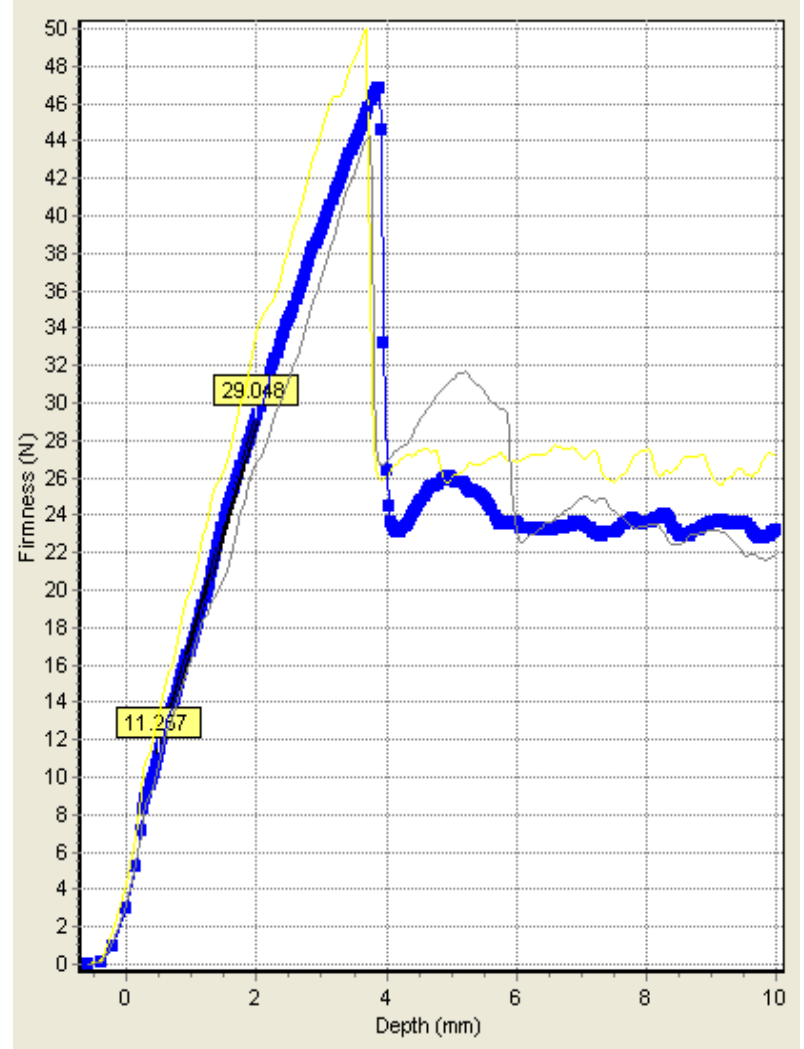

c. alimentary paper packed apple after seven days of storage

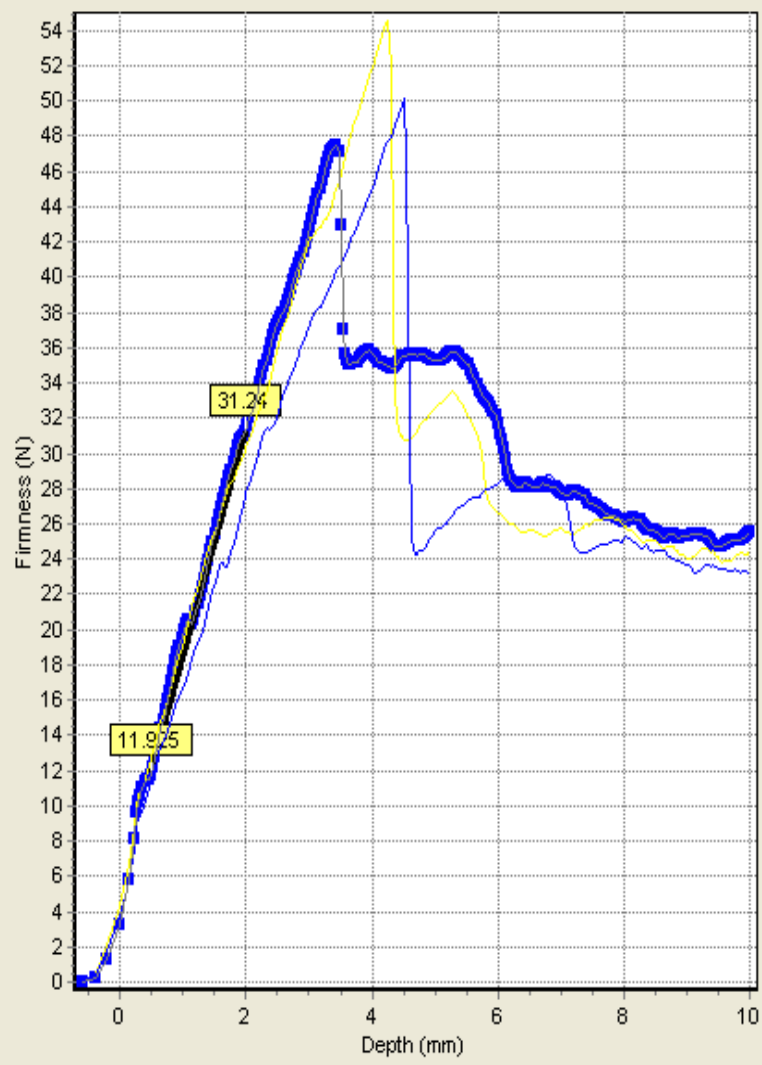

b. apple texture after 15 days of storage

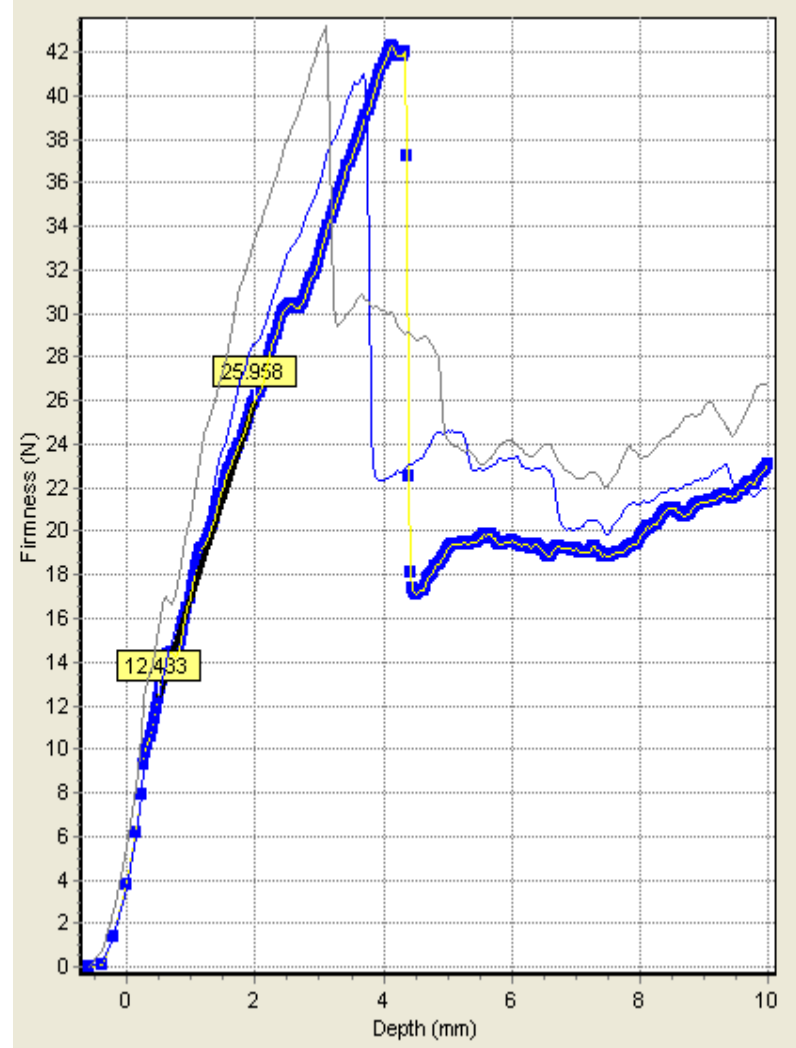

d. alimentary paper packed apple after 15 days of storage 


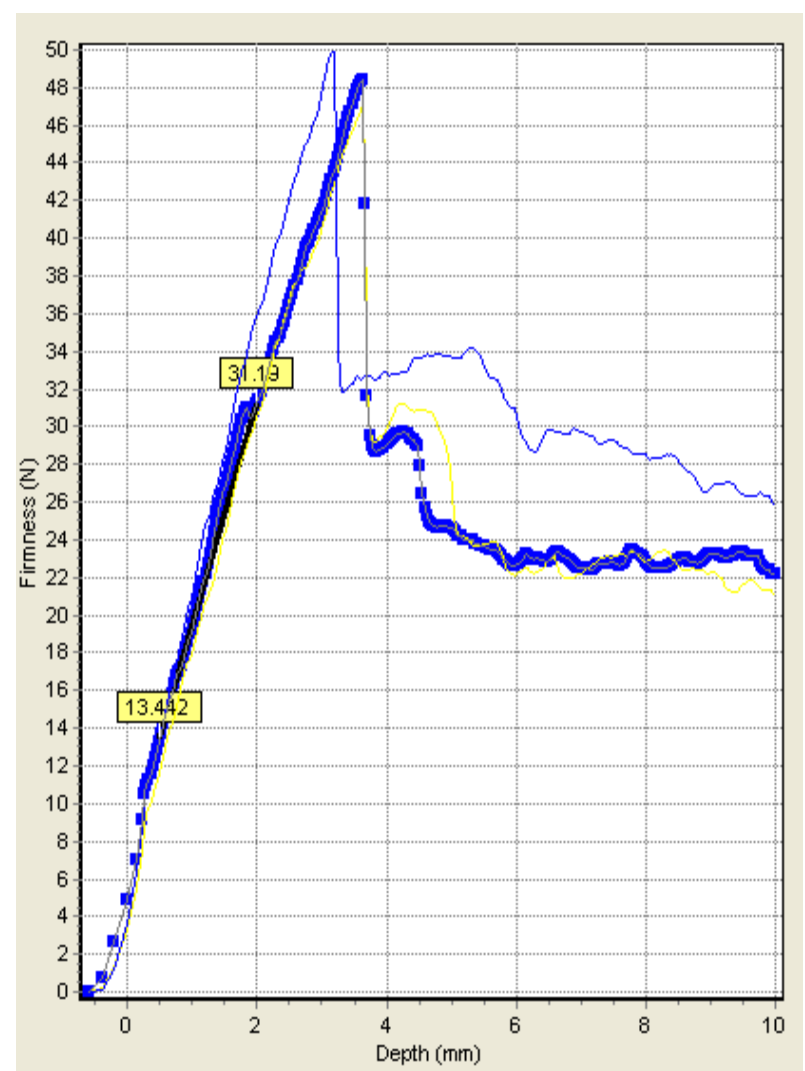

e. LDPE foil packed apple after seven days of storage

Fig. 4 Golden Delicious variety texture curves during storage.

So the LDPE foil is the most suitable type of package used for apples storage at refrigeration temperature, associated with the firmness of the first layer of the apple and the soft and juicy pulp.

\section{Conclusions}

The package, which was the most suitable for this purpose is LDPE foil, while the alimentary paper had been situated at the other pole, generating multiple damages.

The best behavior also from the textural and structural point of view than from the chemical and physical, one is registered by the Golden Delicious variety.

The rheological properties of food materials are quite important since they are used to predict the structure. Selected methodologies existed mainly from the works that have been carried out for years in Dr. Kokini's laboratory. Food materials are complex in structure, they do not consist of just one type of material, but several types of materials with various

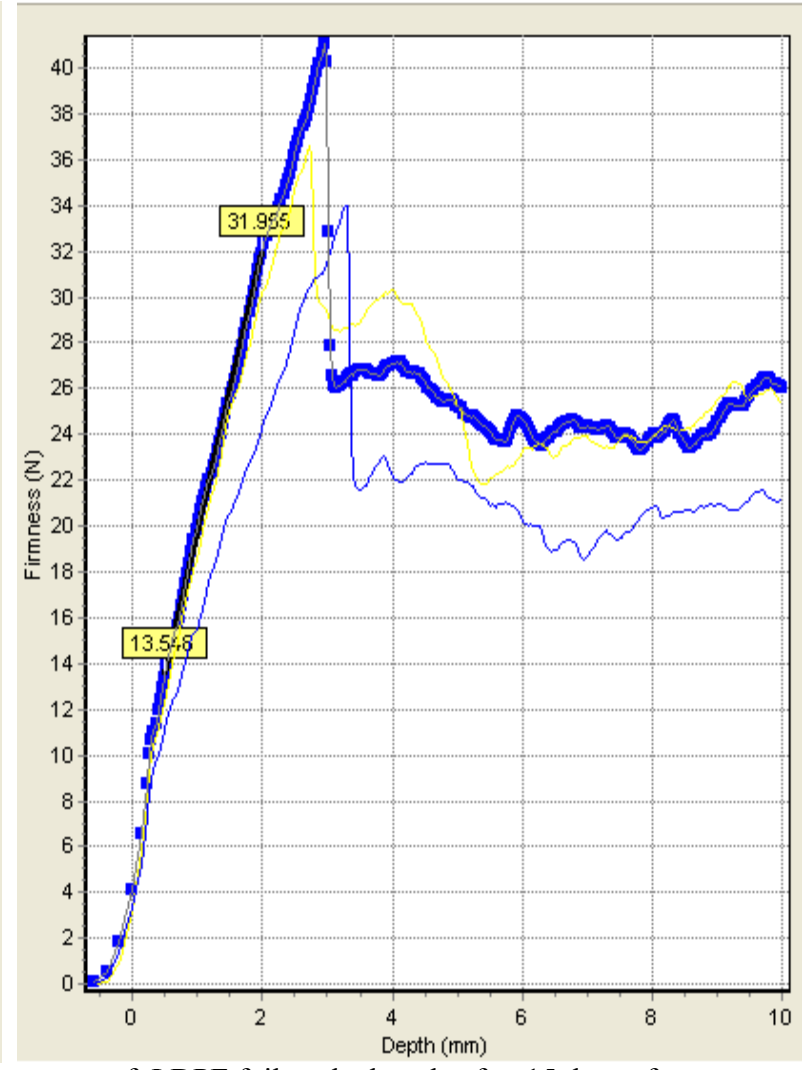

f. LDPE foil packed apple after 15 days of storage

properties like flour, fats and oils, protein, water, sugar, etc., which makes the rheological measurements and simulation of data difficult. Therefore, it is necessary to develop better methods and constitutive models that can predict rheological properties [12].

This study aims at the role of the apple stored by refrigeration and the implications of this preservation process over the textural and structural properties, including the fruits importance as destination the consumers table.

\section{References}

[1] Summary of Noncitrus Fruits and Nuts [Online], http://www.nass.usda.gov/.

[2] Food and Agriculture Organization of the United Nations, Fruit and Vegetable Processing: FAO Agricultural Services Bulletin 119, 1995.

[3] T. Nilsson, Fruit and vegetables quality: An integrated View, Postharvest Handling and Storage of Vegetables, CRC Press, Taylor\&Francis Group, 2000, pp. 96-122.

[4] N.F. Sommer, Postharvest technology of horticultural crops, 
principles of disease suppression by handling practices, Book Publication Division of Agriculture and Natural Resources, University of California, 1992, pp. 109-116.

[5] R.M. Beaudry, Responses of horticultural commodities to low oxygen: Limits to the expanded use of modified atmosphere packaging, Horticulture Technology 10 (3) (2000) 491-500.

[6] J. Ahmed, H. Ramaswamy, S. Kasapis, J.I. Boye, Novel Food Processing Effects on Rheological and Functional Properties, CRC Press, Taylor\&Francis Group, 2010.

[7] E. Mehinagic, G. Royer, R. Symoneaux, F. Jourjon, Relationship between apple sensory attributes and instrumental parameters of texture, Journal of Fruit and Ornamental Plant Research 14 (Suppl. 2), 2006.

[8] I. Shmulevich, N. Galili, M.S. Howarth, Nondestructive impact and acoustic testing for quality assessment of apples, AgEng2002 Conference, Budapest Hungary 30 June-4 July: 02-PH-030.

[9] M.S. Hui, J. Barta, M. Pilar Cano, W. Todd, S. Jiwan Sidhu, K. Nirmal Sinha, Handbook of Fruits and Fruit Processing, Blackwell Publishing, 2006.

[10] Y.H. Hui, F. Chen, P.F. Raquel, M. Guiné, I. Mínguez-Mosquera, L.P. Fernando Pessoa, et al., Handbook of Fruit and Vegetable Flavors, John Willey \& Sons Inc. Publication, 2010.

[11] A.K. Thompson, Fruit and Vegetables: Harvesting, Handling and Storage, Blackwell Publishing, 2003.

[12] Ö. Semih, N. Sozer, L. Kokini Jozef, Rheological instruments in food analysis, in: Handbook of Food Analysis Instruments, CRC Press, Taylor\&Francis Group 2009. 